\title{
Multiparametric Quantum Deformation of the General Linear Supergroup
}

\author{
$\mathrm{Yu}$. I. Manin \\ Steklov Mathematical Institute, Moscow, USSR
}

\begin{abstract}
In the work L. D. Faddeev and his collaborators, and subsequently V. G. Drinfeld, M. Jimbo, S. L. Woronowicz, a new class of Hopf algebras was constructed. They can be considered as one-parametric deformations of either group ring or the universal enveloping algebra of a simple algebraic group. In this paper we define and investigate a multiparametric deformation of the general linear supergroup. This is the simplest example of some general constructions described in $[5,6]$.
\end{abstract}

\section{Introduction}

Quantum groups were recently introduced and studied from various viewpoints in the work of Faddeev and his collaborators (cf. [3] and references therein) followed by Drinfeld [2], Jimbo [4], and Woronowicz [7].

In $[5,6]$ I described a class of quantum groups which are natural symmetries of non-commutative algebraic varieties defined by quadratic equations. This approach furnishes a vast supply of new quantum groups together with their representations.

This paper is devoted to the detailed and explicit study of the simplest groups that can be constructed in this way. It can be read independently of $[5,6]$ although it is useful to keep in mind the underlying philosophy explained in [6]. In addition, we take into account some new effects of a structural $\mathbf{Z}_{2}$-grading thus superizing some parts of $[5,6]$.

Our working definition of a quantum (super)group is that of a Hopf (super)algebra generated by the entries of a multiplicative matrix i.e., admitting a faithful finite-dimensional corepresentation. This viewpoint is close to that of $[3,7]$ but dual to that of $[2,4]$.

The paper is structured as follows. In Sect. 1 we state all essential definitions and results. Proofs and some complements are given in Sects. 2-5.

\section{Notation and Results}

1.1. Hopf Superalgebras and Quantum Supergroups. All our objects are defined over a ground field $k$ of characteristic $\neq 2$ e.g., $\mathbf{R}$ or $\mathbf{C}$. An associative algebra with 
unit is a triple $(E, m, \eta)$ consisting of a linear space $E$, a linear multiplication map $m: E \otimes E \rightarrow E$ and a unit map $\eta: k \rightarrow E$ subject to the well known axioms. A coassociative coalgebra with a counit $(E, \Delta, \varepsilon)$ is defined by inverting all arrows in the diagrams of data and axioms for $(E, m, \eta)$. In particular, we have $\Delta: E \rightarrow E \otimes E$, $\varepsilon: E \rightarrow k$. A bialgebra is a linear space which is simultaneously algebra and coalgebra, both structures being connected by the condition that $\Delta: E \rightarrow E \otimes E$ is an algebra morphism. Finally, a Hopf algebra is a bialgebra endowed with an "antipodal map" $i: E \rightarrow E$ such that

$$
m(i \otimes \mathrm{id}) \Delta=m(\mathrm{id} \otimes i) \Delta=\mathrm{id} .
$$

Bialgebras form a convenient algebraic framework for introducing and studying quantum semigroups, while Hopf algebras do the same for quantum groups.

In order to define superbialgebras, Hopf superalgebras, and eventually, quantum supergroups, one makes the following minor changes in definitions. The space $E$ becomes $\mathbf{Z}_{2}$-graded, and this grading is extended to the tensor algebra of $E$. All maps are grade-preserving. Finally, signs are added in many places e.g., the multiplication in the tensor product of two superalgebras is given by

$$
(a \otimes b)(c \otimes d)=(-1)^{\hat{b} \hat{c}} a c \otimes b d,
$$

where we generally denote by $\hat{b}$ the $\mathbf{Z}_{2}$-degree of $b$.

We shall call a format an arbitrary sequence $\left(a_{1}, \ldots, a_{n}\right), a_{i} \in \mathbf{Z}_{2}$. A matrix $Z=\left(z_{i}^{k}\right)$ with entries in a $\mathbf{Z}_{2}$-graded space has this format if $\hat{z}_{i}^{k}=a_{i}+a_{k}$ (then it has also format $\left.\left(1-a_{1}, \ldots, 1-a_{n}\right)\right)$.

A matrix $Z$ with entries in a superalgebra $(E, \Delta, \varepsilon)$ is called multiplicative one if it has a format and

$$
\begin{aligned}
& \Delta(Z)=Z \otimes Z, \text { i.e. } \Delta\left(z_{i}^{k}\right)=\sum_{j=1}^{n} z_{i}^{j} \otimes z_{j}^{k}, \\
& \varepsilon(Z)=I, \text { i.e. } \varepsilon\left(z_{i}^{k}\right)=\delta_{i}^{k} .
\end{aligned}
$$

1.2. Deformation Parameters and Commutation Relations. From now on we fix a format $\left\{a_{1}, \ldots, a_{n}\right\}$ and a family $q=\left\{q_{i j} \mid 1 \leqq i, j n\right\}$ of non-zero elements of $k$. We put $\hat{i}=a_{i}$ and denote by $E_{q}$ the algebra generated by $n^{2}$ symbols $z_{i}^{k}$ subject to the following relations:

$$
\begin{aligned}
& \left(z_{i}^{k}\right)^{2}=0 \quad \text { for } \hat{i}+\hat{k} \text { odd; } \\
& z_{i}^{k} z_{i}^{l}-(-1)^{(\hat{k}+1)(\hat{l}+1)} q_{k l} z_{i}^{l} z_{i}^{k}=0 \text { for } \hat{i} \text { odd, } k<l ; \\
& z_{i}^{k} z_{i}^{l}-(-1)^{\hat{k} \hat{l}} q_{k l}^{-1} z_{i}^{l} z_{i}^{k}=0 \quad \text { for } \hat{i} \text { even, } k<l ; \\
& z_{i}^{k} z_{j}^{k}-(-1)^{\hat{i} \hat{j}} q_{i j}^{-1} z_{j}^{k} z_{i}^{k}=0 \text { for } \hat{k} \text { even, } i<j ; \\
& z_{i}^{k} z_{j}^{k}-(-1)^{(\hat{i}+1)(\hat{j}+1)} q_{i j} z_{j}^{k} z_{i}^{k}=0 \quad \text { for } \hat{k} \text { odd, } i<j ; \\
& (-1)^{\hat{k}(\hat{j}+\hat{l})} z_{i}^{k} z_{j}^{l}+(-1)^{\hat{j} \hat{l}} q_{k l} z_{i}^{l} z_{j}^{k} \\
& =q_{i j}^{-1}(-1)^{\hat{i} \hat{j}}\left[(-1)^{\hat{k}(\hat{i}+\hat{l})} z_{j}^{k} z_{i}^{l}+(-1)^{\hat{i} \hat{l}} q_{k l} z_{j}^{l} z_{i}^{k}\right] \\
& (-1)^{(\hat{k}+1)(\hat{j}+\hat{l})} z_{i}^{k} z_{j}^{l}+(-1)^{(\hat{j}+1)(\hat{l}+1)} q_{k l}^{-1} z_{i}^{l} z_{j}^{k} \\
& =q_{i j}(-1)^{(\hat{i}+1)(\hat{j}+1)}\left[(-1)^{(\hat{k}+1)(\hat{i}+\hat{l})} z_{j}^{k} z_{i}^{l}\right. \\
& \left.+(-1)^{(\hat{i}+1)(\hat{l}+1)} q_{k l}^{-1} z_{j}^{l} z_{i}^{k}\right] \quad \text { for } i<j, k<l .
\end{aligned}
$$


We define a $\mathbf{Z}_{2}$-grading of $E_{q}$ by $z_{i}^{k}=\hat{i}+\hat{k}$, so that $Z=\left(z_{i}^{k}\right)$ has format $\left(a_{1}, \ldots, a_{n}\right)$. Now we can state our first main result;

1.3. Theorem. There is a unique structure of a superalgebra on $E_{q}$ for which $Z$ is multiplicative.

We make two comments to clarify the statement.

Firstly, if one puts $q_{i j}=1$ for all $i, j$, then (1.3)-(1.9) become equivalent to the simple supercommutation relations $\left[z_{i}^{j}, z_{k}^{l}\right]=0$. Therefore, $E_{q}$ is a deformation of the ring of polynomial functions on the supermanifold $\operatorname{Mat}(a \mid b)$ where a (respectively $b$ ) is the number of even (respectively odd) $a_{i}$ in our format.

Secondly, the Theorem 1.3 is equivalent to the following statement made in the language of "points." Consider a $k$-superalgebra $B$ and two matrices $Z_{1}, Z_{2}$ with elements in $B$ of format $\left(a_{1}, \ldots, a_{n}\right)$. Assume that each element of $Z_{1}$ supercommutes with all elements of $Z_{2}$. Assume also that elements of $Z_{1}$ and $Z_{2}$ separately verify (1.3)-(1.9). Then the same is true for $Z_{1} Z_{2}$. In other words, one can multiply two points of a quantum semigroup if they are "simultaneously observable."

In principle, one can check Theorem 1.3 by a direct calculation. However it would be messy and non-instructive. Instead, we shall deduce it by showing that $E_{q}$ is a universal bialgebra co-acting upon two dual "quantum superspaces" $A_{q}$ and $A_{q}^{*}$.

1.4. Quantum Superspace $A_{q}$. This space or, rather, the polynomial function ring on it is generated by coordinates $x_{1}, \ldots, x_{n}$ with parity assignment $x_{i}=\hat{i}$ and commutation rules

$$
\begin{gathered}
x_{i}^{2}=0 \quad \text { for } \hat{i}=1 ; \\
x_{i} x_{j}-q_{i j}^{-1}(-1)^{\hat{i} \hat{j}} x_{j} x_{i}=0 \text { for } i<j .
\end{gathered}
$$

1.5. Quantum Superspace $A_{q}^{*}$. It is generated by coordinates $\xi^{1}, \ldots, \xi^{n}$ with $\hat{\xi}^{k}=1-\hat{k}$ and commutation rules

$$
\begin{gathered}
\left(\xi^{k}\right)^{2}=0 \quad \text { for } \hat{k}=0 ; \\
\xi^{k} \xi^{1}-q_{k l}(-1)^{(\hat{k}+1)(\hat{l}+1)} \xi^{l} \xi^{k}=0 \text { for } k<l .
\end{gathered}
$$

Note that if we put $V=\oplus k x_{i}, V^{*}=\oplus k \xi^{j}$ and define an $o d d$ bilinear pairing $\langle\mid\rangle: V^{*} \otimes V \rightarrow k$ by $\left\langle\xi^{j} \mid x_{i}\right\rangle{ }^{i} \delta_{i}^{j}$, then the left-hand side tensors in (1.12), (1.13) generate a subspace in $V^{*} \otimes V^{*}$ that is the orthogonal complement of the subspace in $V \otimes V$ generated by the left-hand sides of (1.10), (1.11) [to check it use $\left.\left\langle\xi^{k} \otimes \xi^{1} \mid x_{i} \otimes x_{j}\right\rangle=(-1)^{\hat{i}(\hat{l}+1)} \delta_{i}^{k} \delta_{j}^{l}\right]$.

Therefore, $A_{q}^{*}$ is different from $A_{q}^{!}$as defined in $[5,6]$, where an even bilinear pairing is used. One can say that $*$ combines! and parity change.

We shall deduce Theorem 1.3 from the following result:

1.6. Theorem. a) There exist algebra morphisms

such that

$$
\begin{gathered}
\delta: A_{q} \rightarrow E_{q} \otimes A_{q}, \quad \delta^{*}: A_{q}^{*} \rightarrow E_{q} \otimes A_{q}^{*} \\
\delta(x)=Z \otimes x \text { i.e., } \delta\left(x_{i}\right)=\sum_{j=1}^{n} z_{i}^{j} \otimes x_{j} ; \\
\delta^{*}(\xi)=Z \otimes \xi \text { i.e., } \delta^{*}\left(\xi^{k}\right)=\sum_{k=1}^{n} z_{k}^{l} \otimes \xi^{l} .
\end{gathered}
$$


b) The pair $\left(\delta, \delta^{*}\right)$ is universal in the following sense. Let $B$ be a superalgebra and $\left(\gamma, \gamma^{*}\right)$ two superalgebra morphisms

$$
\gamma: A_{q} \rightarrow B \otimes A_{q}, \quad \gamma^{*}: A_{q}^{*} \rightarrow B \otimes A_{q}^{*}
$$

such that $\gamma(V) \subseteq B \otimes V, \gamma^{*}\left(V^{*}\right) \subseteq B \otimes V^{*}$ and

$$
\gamma(x)=Y \otimes x, \quad \gamma^{*}(\xi)=Y \otimes \xi
$$

for some $Y \in \operatorname{Mat}(n, B)$ of the same format $(\hat{i})$ as $Z$. Then there exists a unique superalgebra morphism $\beta: E_{q} \rightarrow B$ such that

$$
\gamma=(\beta \otimes \mathrm{id}) \delta, \quad \gamma^{*}=(\beta \otimes \mathrm{id}) \delta^{*} .
$$

Theorems 1.3 and 1.6 are proved in Sect. 2.

In the course of deduction of 1.3 from 1.6 it will become clear that $\delta$ and $\delta^{*}$ define corepresentations of the bialgebra $E_{q}$ upon $A_{q}, A_{q}^{*}$ and also upon each homogeneous component of these algebras with respect to the $\mathbf{Z}$-grading $\operatorname{deg}\left(x_{i}\right)$ $=\operatorname{deg}\left(\xi^{j}\right)=1$.

1.7. Quantum Supergroups. Bialgebra $E_{q}$ has no antipode. We interpret the problem of construction of a quantum supergroup from a quantum supersemigroup $E$ as a problem of construction of a universal bialgebra map $E \rightarrow H$, where $H$ is a Hopf superalgebra.

We can give an explicit description of such a map in the case when $E$ is generated by entries of a multiplicative matrix $Z$. In order to do that, define the supertransposed matrix $Z^{\text {st }}$ by

$$
\left(Z^{s t}\right)_{i}^{k}=(-1)^{\hat{k}(\hat{i}+1)}(Z)_{k}^{i} .
$$

Note that if we replace the format of $Z$ by $(1-\hat{i}), Z^{\text {st }}$ will change. Therefore one should rather write $(Z \text {, format })^{s t}$. However, we shall omit format in notation and prescribe to $Z^{s t}$ the same format as to $Z$. We have $(s t)^{4}=\mathrm{id}$ but $(s t)^{2} \neq \mathrm{id}$ in general.

Assume now that a superbialgebra $E$ is generated (as algebra) by the entries of a multiplicative matrix $Z=\left(z_{i}^{j}\right)$. Put $E=k\left\langle\bar{z}_{i}^{j}\right\rangle / R_{0}$, where $\bar{z}_{i}^{j}$ are free associative variables and $R_{0}$ is the ideal of relations between $z_{i}^{j}$. Put $\bar{Z}_{0}=\left(\bar{z}_{i}^{j}\right)$ and introduce an infinite sequence of matrices of the same format $\bar{Z}_{0}, \bar{Z}_{1}, \bar{Z}_{2}, \ldots$ with independent entries. Denote by $\bar{H}$ the free associative algebra generated by these entries.

Define a structure of superbialgebra on $\bar{H}$ by

$$
\bar{\Delta}\left(Z_{k}^{s t^{k}}\right)=\bar{Z}_{k}^{s t^{k}} \otimes \bar{Z}_{k}^{s t^{k}}, \bar{\varepsilon}\left(\bar{Z}_{k}\right)=I ; \quad k=0,1,2, \ldots .
$$

Define a linear map $i: \bar{H} \rightarrow \bar{H}$ by

$$
i\left(\bar{Z}_{k}\right)=\bar{Z}_{k+1}, \quad i(u v)=(-1)^{\hat{i} \hat{v}} i(v) i(u) .
$$

Denote by $\bar{R}$ the ideal in $\bar{H}$ generated by the following sets:

$$
\begin{aligned}
& \text { the entries of } \bar{Z}_{k}^{s t^{k}} \bar{Z}_{k+1}^{s t^{k}}-I, \bar{Z}_{k+1}^{s t^{k}} \bar{Z}_{k}^{s t^{k}}-I ; \\
& \qquad R_{k}=i^{k}\left(R_{0}\right) ; \quad k=0,1,2, \ldots
\end{aligned}
$$

Put $H=\bar{H} / \bar{R}$. Clearly, there exists a superalgebra morphism $\gamma: E \rightarrow H$ defined by $\gamma(Z)=\bar{Z}_{0} \bmod \bar{R}_{0}$. 
1.8. Theorem. a) $\bar{R}$ is a coideal in $\bar{H}$. Therefore, $\bar{\Delta}$ induces on $H$ a superbialgebra structure and $\gamma$ is a superbialgebra morphism.

b) $i(\bar{R}) \subset \bar{R}$, and $i$ induces upon $H$ an antipodal map so that $H$ is a Hopf superalgebra.

c) For an arbitrary superbialgebra morphism $\gamma^{\prime}: E \rightarrow H^{\prime}$, where $H^{\prime}$ is a Hopf superalgebra, there exists a unique Hopf superalgebra morphism $\beta: H \rightarrow H^{\prime}$ such that $\gamma^{\prime}=\beta \gamma$.

If we apply this construction to $E_{q}$ we shall obtain the Hopf superalgebra $H_{q}$ which is our "general linear quantum supergroup."

This theorem is proved in Sect. 3.

1.9. Quantum Determinant. As was explained in $[5,6]$, there is a natural construction of the quantum determinant of the matrix $Z$ generating $E_{q}$ when it is of the format $(1, \ldots, 1)$ i.e., when $A_{q}$ is a deformed exterior algebra of $n$ indeterminates $x_{1}, \ldots, x_{n}$. We simply define $D=\operatorname{DET}_{q}(Z) \in E_{q}$ by the identity

$$
\Delta\left(x_{1} \ldots x_{n}\right)=D \otimes x_{1} \ldots x_{n} \in E_{q} \otimes A_{q},
$$

which exists since $x_{1} \ldots x_{n}$ generates the degree $n$ component of $A_{q}$.

From this definition one sees that $\Delta(D)=D \otimes D$, and

$$
\operatorname{DET}_{q}(Z)=\sum \operatorname{sgn}\left(i_{1}, \ldots, i_{n}\right) \prod_{\substack{a>b \\ i_{a}<i_{b}}} q_{i_{a} i_{b}} z_{1}^{j_{1}} \ldots z_{n}^{j_{n}} .
$$

This definition is based upon the existence of a natural one-dimensional comodule for $E_{q}$, the quantum highest exterior power $\left(A_{q}\right)_{n}$ of the fundamental corepresentation.

In the general case, it is necessary to construct the quantum Berezinian. Since it is not polynomial in entries of $Z$ even in the classical case, one cannot expect it to lie in $E_{q}$. It may lie in $H_{q}$ where $Z$ becomes invertible. However, in order to construct the one-dimensional corepresentation furnishing our Berezinian I shall have to diminish $H_{q}$ further making an additional factorization. I do not know whether it is really necessary.

1.10. Quantum Koszul Complex. Put $B_{q}=A_{q}^{*} \otimes A_{q}$. Clearly, this is an algebra of the same kind as $A_{q}$, with generators $\left(\xi^{1} \otimes 1, \ldots, \xi^{n} \otimes 1,1 \otimes x_{1}, \ldots, 1 \otimes x_{n}\right)$ of format $\left(1-a_{1}, \ldots, 1-a_{n}, a_{1}, \ldots, a_{n}\right)$. Put

$$
c=\sum_{i=1}^{n} \xi^{i} \otimes x_{i} \in B_{q} .
$$

One immediately verifies that $c^{2}=0$. Let $d: B_{q} \rightarrow B_{q}$ be the linear map $d f=f c$. Put $H^{\prime}\left(B_{q}\right)=\operatorname{Ker}(d) / \operatorname{Im}(d)$.

1.11. Proposition. $H^{\prime}\left(B_{q}\right)$ is an one-dimensional k-space generated by

$$
\prod_{i=0} \xi^{i} \prod_{j=1} x_{j} \bmod \operatorname{Im}(d) \text {. }
$$

This space will be our superanalog of the highest exterior power. Now we must prepare a Hopf superalgebra coacting upon it. 
Let us start with the universal Hopf algebra $G_{q}$ coacting upon $B_{q}$, $\delta: B_{q} \rightarrow G_{q} \otimes B_{q}$. Put

$$
\delta\left(\begin{array}{c}
\xi \\
x
\end{array}\right)=\left(\begin{array}{cc}
U & W \\
W^{\prime} & V
\end{array}\right) \otimes\left(\begin{array}{c}
\xi \\
x
\end{array}\right) ; \quad \xi=\left(\begin{array}{c}
\xi^{1} \\
\vdots \\
\xi^{n}
\end{array}\right), \quad x=\left(\begin{array}{c}
x_{1} \\
\vdots \\
x_{n}
\end{array}\right) .
$$

1.12. Theorem. a) The entries of the matrices $i^{n}(W), i^{n}\left(W^{\prime}\right)$ and $i^{n}\left(V-i\left(U^{s t}\right)\right)$, $n=0,1,2, \ldots$ generate an $i$-stable coideal $S$ in $G_{q} . P u t F_{q}=G_{q} / S$ with natural coaction upon $B_{q}$ which we denote $\delta_{F}$.

b) $\delta_{F}(c)=1 \otimes c \in F_{q} \otimes B_{q}$. Therefore, $\delta_{F}$ induces a coaction

$$
\delta_{H}: H^{\prime}\left(B_{q}\right) \rightarrow H^{\prime}\left(F_{q} \otimes B_{q}\right)=F_{q} \otimes H^{\prime}\left(B_{q}\right) .
$$

c) Put

$$
\delta_{H}\left(\prod_{i=0} \xi^{i} \prod_{j=1} x_{j}\right)=D \otimes \prod_{i=0} \xi^{i} \prod_{j=1} x_{j} \bmod \operatorname{Im}(d) .
$$

Then $\delta_{H}(D)=D \otimes D ; D$ is called the quantum Berezinian (of $V$ ).

Note that in view of the universality, there exists a Hopf superalgebra morphism $H_{q} \rightarrow F_{q}$ for which $Z$ goes into $V$. If it is an embedding we may transport $D$ into $H_{q}$.

1.13. The Structure of $E_{q}$. It is pretty clear that $A_{q}$ (respectively $A_{q}^{*}$ ) is "of the same size" as a polynomial ring of a even and $b$ odd (respectively $a$ odd and $b$ even) supercommuting variables. This means that the normally ordered monomials, say, $x_{1}^{m_{1}} \ldots x_{n}^{m_{n}}$, with $m_{i}=0,1,2, \ldots$ for $\hat{i}=0$ and $m_{i}=0,1$ for $\hat{i}=1$ form a $k$-basis of $A_{q}$.

It is known that a similar statement is true for $E_{q}$ when all $q_{i j}$ are equal and there are no odd variables (in the dual language, this is equivalent to the quantum Poincaré-Birkhoff-Witt theorem). On the other hand, it is false for general values of the parameters. We prove the following result which falls short of giving the complete picture.

Define an ordering of $z_{i}^{j}$ by

$$
z_{i}^{j}<z_{k}^{l} \text { if either } i>k \text {, or } i=k, j>l .
$$

Call a monomial in $z_{i}^{j}$ normally ordered if for any $z^{\prime}<z^{\prime \prime}$ in this monomial $z^{\prime}$ is to the left of $z^{\prime \prime}$, and if no odd $z^{\prime}$ enters this monomial twice.

1.14. Theorem. a) Quadratic normally ordered monomials form a basis of the quadratic part of $E_{q}$ iff $q_{i j}^{2} \neq-1$ for all $i, j$.

b) If this condition is satisfied then the normally ordered monomials span $E_{q}$. They are linearly independent iff cubic monomials are independent, and this can be true only if $q_{i j}= \pm q \pm 1$ for some $q$. For $n=2$ this is always true.

Note that a specialization of the values of $q_{i j}$ may enlarge $E_{q}$. Somewhat paradoxically, the largest $E_{q}$ for $n=2$ corresponds to $q^{2}=-1$. Therefore, from the Hopf viewpoint, the algebra $k\langle x, y\rangle /(x y-i y x\rangle$ is more symmetrical then $k[x, y]$ ! 


\section{Proof of Theorems $\mathbf{1 . 3}$ and $\mathbf{1 . 6}$}

2.1. Proof of the Theorem 1.6. We start with an arbitrary superalgebra morphism $\gamma: A_{q} \rightarrow B \otimes A_{q}$, linear in $x_{i}$, and temporarily denote by $z_{i}^{k} \in B$ the coefficients in

$$
\gamma\left(x_{i}\right)=\sum_{j=1}^{n} z_{i}^{j} \otimes x_{j} .
$$

Clearly, $\gamma$ is well defined iff it conserves the identities (1.10), (1.11). We must apply $\gamma$ to the left-hand side of these identities and then calculate the coefficients of the resulting expressions represented as linear combinations of monomials $1 \otimes x_{i} x_{j}$, $i<j$, which are linearly independent over $B$ in $B \otimes A_{q}$. In this way we shall obtain one half of the commutation relations (1.3)-(1.9), namely:

$$
\begin{aligned}
& \text { coefficient of } x_{k}^{2}, \hat{k}=0, \text { in } \gamma(1.10) \Rightarrow(1.3) \text { for } \hat{i}=1, \hat{k}=0 ; \\
& \text { coefficient of } x_{k} x_{1}, k<l, \hat{i}=1 \text { in } \gamma(1.10) \Rightarrow(1.4) ; \\
& \text { coefficient of } x_{k}^{2}, \hat{k}=0 \text {, in } \gamma(1.11) \Rightarrow(1.6) ; \\
& \text { coefficient of } x_{k} x_{l}, k<l \text {, in } \gamma(1.11) \Rightarrow(1.8) .
\end{aligned}
$$

The other half of our relations is supplied by $\gamma^{*}: A_{q}^{*} \rightarrow B \otimes A_{q}^{*}$. It can be directly deduced from the previously established relations applying to them the parity charge $\hat{i} \rightarrow 1-\hat{i}$ and the parameter change $q_{i j} \rightarrow q_{i j}^{-1}$.

This calculation proves simultaneously both parts of Theorem 1.6.

2.2. Proof of the Theorem 1.3. Apply now the universality statement of Theorem 1.6 to the following situation: $B=E_{q} \otimes E_{q}$, and

$$
\begin{gathered}
\gamma=(\mathrm{id} \otimes \delta) \delta: A_{q} \rightarrow E_{q} \otimes E_{q} \otimes A_{q} ; \\
\gamma^{*}=\left(\mathrm{id} \otimes \delta^{*}\right) \delta^{*}: A_{q} \rightarrow E_{q} \otimes E_{q} \otimes A_{q}^{*} .
\end{gathered}
$$

We get the map $\beta$ denoted now $\Delta: E_{q} \rightarrow E_{q} \otimes E_{q}$. From its definition it follows that $\Delta(Z)=Z \otimes Z$, that $\Delta$ is coassociative and that $\varepsilon: Z \rightarrow I$ is a counit. One also checks directly that $\delta$ (respectively $\delta^{*}$ ) defines on $A_{q}$ (respectively on $A_{q}^{*}$ ) the structure of a left comodule.

2.3. Fundamental Corepresentations and Their Symmetric Powers. For each $m \geqq 0$, the space of forms of degree $m$ in $A_{q}$ plays a role of the $m^{\text {th }}$ symmetric power of the fundamental corepresentation $V=\oplus k x_{i}$. Similarly, we have the "symmetric quantum powers" of the odd-contragradient corepresentation $V^{*}=\oplus k \xi^{j}$.

\section{Proof of the Theorem 1.8}

3.1. Lemma. a) Let $Z$ be a matrix with entries in a supercoalgebra $(E, \Delta, \varepsilon)$ which has a format. Then

$$
\Delta(Z)=Z \otimes Z \Leftrightarrow \Delta^{\mathrm{op}}\left(Z^{\mathrm{st}}\right)=Z^{\mathrm{st}} \otimes Z^{\mathrm{st}},
$$

where

$$
\Delta^{\mathrm{op}}=S_{12} \Delta, \quad S_{12}(a \otimes b)=(-1)^{\hat{a} \hat{b}} b \otimes a .
$$


b) Let $Z$ be a multiplicative matrix in a Hopf superalgebra. Put $Z_{k}=i^{k}(Z)$. Then $Z_{k}^{\mathrm{st}^{k}}$ is multiplicative, and

$$
Z_{k}^{\mathrm{st}^{k}} Z_{k+1}^{\mathrm{st}^{k}}=Z_{k+1}^{\mathrm{st}^{k}} Z_{k}^{\mathrm{st}^{k}}=I .
$$

Proof. a. Let $Z=\left(z_{i}^{k}\right), \Delta(Z)=Z \otimes Z$. Then

$$
\begin{aligned}
\left(\Delta^{\mathrm{op}}\left(Z^{\mathrm{st}}\right)\right)_{i}^{k} & =(-1)^{\hat{k}(\hat{i}+1)} \Delta^{\mathrm{op}}(Z)_{k}^{i}=(-1)^{\hat{k}(\hat{i}+1)} S_{12}\left(\sum_{j} z_{k}^{j} \otimes z_{j}^{i}\right) \\
& =(-1)^{\hat{k}(\hat{i}+1)} \sum_{j}(-1)^{(\hat{k}+\hat{j})(\hat{i}+\hat{j})} z_{j}^{i} \otimes z_{k}^{j}, \\
\left(Z^{\mathrm{st}} \otimes Z^{\mathrm{st}}\right)_{i}^{k} & =\sum_{j}\left(Z^{\mathrm{st}}\right)_{i}^{j} \otimes\left(Z^{\mathrm{st}}\right)_{j}^{k}=\sum_{j}(-1)^{\hat{j}(\hat{i}+1)} z_{j}^{i} \otimes(-1)^{\hat{k}(\hat{j}+1)} z_{k}^{j} .
\end{aligned}
$$

Comparing signs, we get the implication $\Rightarrow$ in (3.1). The inverse implication is similar.

b. Applying the antipode axiom (1.1) to $Z$ and using the multiplicativity we get

$$
i(Z) Z=Z i(Z)=I,
$$

i.e., (3.2) for $k=0$. Now, we have $i m=m S_{12}(i \otimes i)$ (see [1] for the non-graded case). Calculating as in a. we get from here that $i\left((A B)^{\mathrm{st}}\right)=i\left(B^{\mathrm{st}}\right) i\left(A^{\mathrm{st}}\right)$ if $A, B$ are of the same format. Applying $i$ to (3.2) $)^{\text {st }}$ we get (3.2) with $k+1$ instead of $k$.

Finally, again as in a., we have $S_{12}(Z \otimes Z)^{\text {st }}=Z^{\text {st }} \otimes Z^{\text {st }}$. Hence, assuming that $Y=Z_{k}^{\mathrm{st}^{k}}$ is multiplicative, we can prove that $Z_{k+1}^{\mathrm{st}^{k+1}}=i\left(Y^{\mathrm{st}}\right)$ is multiplicative using the identity $\Delta i=S_{12}(i \otimes i) \Delta$ (cf. again [1] for the non-graded case):

$$
\Delta i\left(Y^{\mathrm{st}}\right)=S_{12}(i \otimes i) \Delta\left(Y^{\mathrm{st}}\right)=S_{12}(i(Y) \otimes i(Y))^{\mathrm{st}}=i(Y)^{\mathrm{st}} \otimes i(Y)^{\mathrm{st}} .
$$

3.2. $\bar{R}$ is a Coideal. Since $E$ is a coalgebra with $\Delta(Z)=Z \otimes Z$, we have $\bar{\Delta}\left(R_{0}\right)$ $c k\left\langle\bar{z}_{i}^{j}\right\rangle \otimes R_{0}+R_{0} \otimes k\left\langle\bar{z}_{i}^{j}\right\rangle$. It follows that the same is true for $R_{j}$ with even $j$ instead of $R_{0}$. One can treat odd $j$ similarly using $\left(E, m^{\mathrm{op}}, \Delta^{\mathrm{op}}\right)$ instead of $(E, m, \Delta)$.

In order to treat (1.16) put $A=\bar{Z}_{k}^{\mathrm{st}^{k^{+1}}}, B=\bar{Z}_{k+1}^{\mathrm{st}^{\mathrm{t}^{k}}}$. According to the Lemma 3.1

$$
\bar{\Delta}(A)=A \otimes A, \bar{\Delta}\left(B^{\mathrm{st}}\right)=B^{\mathrm{st}} \otimes B^{\mathrm{st}} .
$$

Hence

$$
\begin{aligned}
& \bar{\Delta}(A B)_{i}^{k}=\left[(A \otimes A)\left(B^{\mathrm{st}} \otimes B^{\mathrm{st}}\right)^{\mathrm{st}}\right]_{i}^{k} \\
& =\sum_{j}(A \otimes A)_{i}^{j}\left(B^{\mathrm{st}} \otimes B^{\mathrm{st}}\right)_{k}^{j}(-1)^{\hat{j}(\hat{k}+1)} \\
& =\sum_{j r s}\left(a_{i}^{r} \otimes a_{r}^{j}\right)\left((-1)^{\hat{s}(\hat{k}+1)} b_{s}^{k} \otimes(-1)^{\hat{j}(\hat{s}+1)} b_{j}^{s}\right)(-1)^{\hat{j}(\hat{k}+1)} \\
& =\sum_{j r s} a_{i}^{r} b_{s}^{k} \otimes a_{r}^{j} b_{j}^{s}(-1)^{(\hat{r}+\hat{j})(\hat{s}+\hat{k})+\hat{s}(\hat{k}+1)+\hat{j}(\hat{s}+1)+\hat{j}(\hat{k}+1)} \\
& =\sum_{r s}(-1)^{\hat{r} \hat{s}+\hat{r} \hat{k}+\hat{s} \hat{k}+\hat{s}} a_{i}^{r} b_{s}^{k} \otimes(A B)_{r}^{s} .
\end{aligned}
$$

From this we deduce

$$
\begin{aligned}
\bar{\Delta}(A B-I)_{i}^{k}= & \sum_{r s}(-1)^{\hat{r} \hat{s}+\hat{r} \hat{k}+\hat{s} \hat{k}+\hat{s}} a_{i}^{r} b_{s}^{k} \otimes\left[(A B)_{r}^{s}-\delta_{r}^{s}\right] \\
& +\sum_{r}\left(a_{i}^{r} b_{r}^{k}-\delta_{i}^{k}\right) \otimes 1
\end{aligned}
$$


which shows that the entries of $A B-I$ generate a coideal. A similar calculation can be applied to $B A-I$.

3.3. $\bar{R}$ is $i$-Stable. Clearly, $i\left(R_{k}\right)=R_{k+1}$. Furthermore, since $i$ reverses multiplication we have $i\left((A B)^{\mathrm{st}}\right)=i\left(B^{\mathrm{st}}\right) i\left(A^{\mathrm{st}}\right)$ so that

$$
i\left(\bar{Z}_{k}^{\mathrm{st}^{k}} \bar{Z}_{k+1}^{\mathrm{st}^{k}}-I\right)=\left(\bar{Z}_{k+2}^{\mathrm{s} \mathrm{s}^{k+1}} \bar{Z}_{k+1}^{\mathrm{s}^{k+1}}-I\right)^{\mathrm{st}^{3}} .
$$

3.4. $i \bmod \bar{R}$ is an Antipode. The entries of $\bar{Z}_{k}^{\mathrm{st}}, k=0,1,2, \ldots \bmod \bar{R}$ generate $H=\bar{H} / \bar{R}$ as a $k$-algebra. Reducing (1.16) $\bmod \bar{R}$ we see that applying (1.1) to any of these generators we get an identity. Therefore, it suffices to check that if (1.1) is verified when applied to $u, v \in H$ it is also verified for $u v$. In fact,

$$
\begin{aligned}
m(i \otimes \mathrm{id})(\Delta(u) \Delta(v)) & =m(i \otimes \mathrm{id})\left(\sum_{k} u_{k}^{\prime} \otimes u_{k}^{\prime \prime}\right)\left(\sum_{l} v_{l}^{\prime} \otimes v_{l}^{\prime \prime}\right) \\
& =m\left(\sum_{k l}(-1)^{\hat{u}_{k}^{\prime} \hat{v}_{l}^{\prime}+\hat{u}_{k}^{\prime} \hat{v}^{\prime}} i\left(v_{l}^{\prime}\right) i\left(u_{k}^{\prime}\right) \otimes u_{k}^{\prime \prime} v_{l}^{\prime \prime}\right) \\
& =\sum_{l} i\left(v_{l}^{\prime}\right)\left(\sum_{k}(-1)^{\hat{\hat{v}} \hat{v}^{\prime}} i\left(u_{k}^{\prime}\right) u_{k}^{\prime \prime}\right) v_{l}^{\prime \prime} \\
& =\eta \varepsilon(u)\left(\sum i\left(v_{l}^{\prime}\right) v_{l}^{\prime \prime}(-1)^{\hat{u} \hat{v} \hat{v}_{l}}\right)=\eta \varepsilon(u) \eta \varepsilon(v) .
\end{aligned}
$$

The last equality is valid even if $\hat{u}=1$ because then $\varepsilon(u)=0$.

3.5. Universality. Let $\gamma^{\prime}: E \rightarrow\left(H^{\prime}, i^{\prime}\right)$ be a superbialgebra morphism to a Hopf superalgebra. Put $Z_{k}^{\prime}=\left(i^{\prime}\right)^{k}\left(\gamma^{\prime}(Z)\right)$. In view of Lemma 3.1, the entries of $Z_{k}^{\prime}$ verify the relations (1.16) and (1.17). This allows us to define $\beta$.

3.6. Bijective and Unipotent Antipodes. The class of Hopf superalgebras with a bijective antipode is closer to that of usual algebraic groups. It is not difficult to modify our construction so as to obtain a universal map $E \rightarrow H$ in this class. It suffices to introduce matrices $\bar{Z}_{k}$ and relations (1.16), (1.17) for all integers $k$.

If we impose additional relations $\bar{Z}_{k}=\bar{Z}_{k+2 d}$ we shall obtain a universal map into a Hopf superalgebra with the antipode verifying $i^{2 d}=\mathrm{id}$.

\section{Quantum Berezinian}

4.1. Cohomology of the Koszul Complex. Since $x_{i}$, $\xi^{j}$ verify (1.10)-(1.13) one easily sees that

$$
\left(\xi^{i} \otimes x_{i}\right)^{2}=0 ;\left(\xi^{i} \otimes x_{i}\right)\left(\xi^{j} \otimes x_{j}\right)+\left(\xi^{j} \otimes x_{j}\right)\left(\xi^{i} \otimes x_{i}\right)=0 ;
$$

hence $c^{2}=0$ and even $c_{\lambda}^{2}=0$ for $c_{\lambda}=\sum \lambda_{i} \xi^{i} \otimes x_{i}, \lambda_{i} \in k$. For $n=1, B_{q}$ is a polynomial ring in one even and one odd variable so that Proposition 1.12 is evident. The general case can be reduced to this one because $B_{q}$ as a complex is isomorphic to the tensor product of $n$ classical $n=1 \mathrm{Koszul}$ complexes. We leave to the reader taking care of twisting by $q_{i j}$.

We now turn to the proof of Theorem 1.12.

4.2. $S$ is a Coideal. We have

$$
\Delta\left(\begin{array}{cc}
U & W \\
W^{\prime} & V
\end{array}\right)=\left(\begin{array}{cc}
U & W \\
W^{\prime} & V
\end{array}\right) \otimes\left(\begin{array}{cc}
U & W \\
W^{\prime} & V
\end{array}\right) .
$$


Hence

$$
\Delta(W)=W \otimes V+U \otimes W ; \quad \Delta\left(W^{\prime}\right)=W^{\prime} \otimes U+V \otimes W^{\prime},
$$

so that the entries of $W$ and $W^{\prime}$ generate a coideal. Applying first to this matrix $i^{n} \mathrm{st}^{n}$ we get the same conclusion for $i^{n}(W), i^{n}\left(W^{\prime}\right)$.

If $A, B$ are two multiplicative matrices of the same format we have

$$
\Delta(A-B)=A \otimes A-B \otimes B=A \otimes(A-B)+(A-B) \otimes B .
$$

Applying this to $A=i^{n}\left(V^{\mathrm{st}^{n}}\right), B=i^{n+1}\left(V^{\mathrm{st}^{n+1}}\right)$ we see that $S$ is a coideal.

4.3. $c$ is a Coinvariant. Put $\xi^{t}=\left(\xi^{1}, \ldots, \xi^{n}\right)$. We have

$$
\delta_{F}\left(\xi^{t}\right)=(U \otimes \xi+W \otimes x)^{t} \bmod S=\xi^{t} \otimes U^{\mathrm{st}} \bmod S=\xi^{t} \otimes i(V) \bmod S .
$$

Therefore

$$
\delta(c)=\delta\left(\xi^{t} x\right)=\xi^{t} \otimes i(V) V \bmod S \otimes x=1 \otimes c .
$$

The rest is clear.

\section{The Structure of $E_{q}$}

5.1. Commutation Relations. The relations (1.3)-(1.9) for fixed indices $i, j, k, l$ constitute a block corresponding to a choice of a two-by-two submatrix in $Z$. In order to present them in a more manageable form we first put

$$
\left(\begin{array}{ll}
z_{i}^{k} & z_{i}^{l} \\
z_{j}^{k} & z_{j}^{l}
\end{array}\right)=\left(\begin{array}{ll}
a & b \\
c & d
\end{array}\right) ; q_{i j}=q_{v}(\text { vertical }) ; q_{k l}=q_{h} \text { (horizontal) } .
$$

Then (1.4)-(1.9) take form

$$
\begin{aligned}
& a b=(-1)^{\hat{a} \hat{b}} q_{h}^{(-1)^{\hat{i}+1}} b a ; \quad c d=(-1)^{\hat{c} \hat{d}} q_{h}^{(-1)^{\hat{j}+1} d c} \\
& a c=(-1)^{\hat{a} \hat{c}} q_{v}^{(-1)^{\hat{k}+1}} c a ; \quad b d=(-1)^{\hat{b} \hat{d}} q_{v}^{(-1)^{\hat{\imath}+1}} d b ; \\
& a d-(-1)^{\hat{a} \hat{a}} q_{v}^{-1} q_{h} d a=-\eta\left(q_{h} b c-(-1)^{\hat{b} \hat{c}} q_{v}^{-1} c b\right) ; \\
& a d-(-1)^{\hat{a} \hat{d}} q_{v} q_{h}^{-1} d a=\eta\left(q_{h}^{-1} b c-(-1)^{\hat{b} \hat{c}} q_{v} c b\right),
\end{aligned}
$$

where $\eta=(-1)^{\hat{k} \hat{j}+\hat{k} \hat{l}+\hat{j} \hat{l}}$. In particular, if $q_{v}=q_{h}=1$ then $a, b, c, d$ pairwise supercommute.

5.2. Quadratic Monomials. Call a monomial $d^{\delta} c^{\gamma} b^{\beta} a^{\alpha}$ normally ordered one if $\alpha, \beta, \gamma, \delta=0,1,2, \ldots$ (respectively 0,1$)$ for even (respectively odd) entries. Four nonnormally ordered monomials $a b, c d, a c, c d$ can be expressed via normally ordered ones with the help of (5.1), (5.2). The rest of them $a d, b c$ can be expressed via (5.3), (5.4) iff

$$
\operatorname{det}\left(\begin{array}{cc}
1 & \eta q_{h} \\
1 & -\eta q_{h}^{-1}
\end{array}\right)=-\eta\left(q_{h}^{-1}+q_{h}\right) \neq 0
$$

i.e., iff $q_{h}^{2} \neq-1$. Otherwise, subtracting (5.3) from (5.4) we get a linear relation between normally ordered monomials

$$
(-1)^{\hat{a} \hat{d}}\left(q_{v}+q_{v}^{-1}\right) q_{h} d a=-(-1)^{\hat{b} \hat{c}} \eta\left(q_{v}+q_{v}^{-1}\right) c b
$$


which disappears if also $q_{v}^{2}=-1$. But then (5.3) and (5.4) become equivalent so that the normally ordered quadratic monomials cease to span the quadratic part of $E_{q}$. In fact, one should complement them by either $a d$ or $b c$.

In general, if $q_{h}^{2} \neq-1,(5.3)$ and (5.4) together are equivalent to

$$
\begin{aligned}
& a d=\frac{q_{v}^{-1}+q_{v}}{q_{h}^{-1}+q_{h}}(-1)^{\hat{a} \hat{d}} d a+\frac{q_{h}^{-1} q_{v}^{-1}-q_{h} q_{v}}{q_{h}^{-1}+q_{h}} \eta(-1)^{\hat{b} \hat{c}} c b, \\
& b c=\frac{q_{v}^{-1}+q_{v}}{q_{h}^{-1}+q_{h}}(-1)^{\hat{c} \hat{b}} c b+\frac{q_{v}^{-1} q_{h}-q_{v} q_{h}^{-1}}{q_{h}^{-1}+q_{h}} \eta(-1)^{\hat{a} \hat{d}} d a .
\end{aligned}
$$

Note now that the normal ordering of entries of $Z$ induces the normal ordering of entries of an arbitrary two-by-two submatrix of $Z$. Therefore, if $q_{i j}^{2} \neq-1$ for all $q_{i j}$, the normally ordered quadratic monomials form a basis of the quadratic part of $E_{q}$. This proves the first assertion of Theorem 1.14.

5.3. Normally Ordered Monomials Span $E_{q}$. Now we shall describe an algorithm $I_{m}, m \leqq n$, allowing one to reduce any polynomial of $z_{i}^{j}, i \geqq m$, to a normal form, i.e., to present it as a linear combination of the normally ordered monomials. We shall do this inductively, starting with $I_{n}$ and then defining consecutively $I_{n-1}$, $I_{n-2}, \ldots, I_{1}$. The last algorithm $I_{1}$ will do the job.

Each algorithm consists in applying a series of "elementary transformations." An elementary transformation substitutes a left-hand side of one of the relations (5.1), (5.2), (5.5), (5.6) occurring in a polynomial by the corresponding right-hand side.

$I_{n}$ acts upon polynomials depending only on the entries of the last line of $Z$. Since the corresponding commutation relations have the simple form (5.1) one can simply rearrange the entries in the normal order supplementing the twisting coefficients and deleting all monomials where a square of an odd variable occurs.

If $I_{m}$ is already defined, $I_{m-1}$ acts in two stages. At the first stage, we take an arbitrary neighbouring pair in a monomial, of the type $y x$, where $y$ lies in the $(m-1)$-th line while $x$ lies below this line. Using (5.5), (5.6) or (5.2) we replace this pair by a linear combination of two pairs of the type $x^{\prime} y^{\prime}$, where $y^{\prime}$ lies in the $(m-1)$-th line while $x^{\prime}$ lies below. After a finite number of such steps we shall arrive at a linear combination of monomials in each of which the elements of the $(m-1)$-th line lie to the right of the elements of the lines $m, m+1, \ldots, n$. At the second stage, we rearrange separately the elements of the $(m-1)$-th line and the rest using respectively $(5.1)$ and $I_{m-1}$.

This algorithm, for the case of pure even format and all $q_{i j}$ equal, was suggested by Yu. Kobyzev.

5.4. Cubic Monomials. Elementary transformations in general can be applied to a polynomial in various orders. If two different normalization procedures lead to different results we get a linear relation between normally ordered monomials in $E_{q}$.

This may first happen in degree three. Namely, a monomial $u v w$ can be normalized in two different ways iff we have $u>v>w$ so that we can start rearranging either $u v$, or $v w$. The following lemma deals with the case when $u, v, w$ lie simultaneously in a two-by-two submatrix of $Z$ as in the beginning of this section. 
5.5. Lemma. Suppose that $q_{h}^{2} \neq-1, q_{v}^{2} \neq-1$. Then the normally ordered cubic monomials in $a, b, c, d$ are linearly independent in $E_{q}$ iff $q_{v}= \pm q_{h}^{+1}$.

Proof. We must directly normalize eight monomials $a^{2} d, a d^{2}, b^{2} d, b d^{2}, a b c, a b d$, $a c d, b c d$ in two different ways and compare the results. We shall do that for $a b c$ and leave the rest to the reader.

Denote by $R_{i j}$ the application of an elementary transformation to the $i j$-places of a monomial e.g., $R_{23}(a b c)=a$ (right-hand side of (5.6)).

Applying $R_{23} R_{12} R_{23}$ to $a b c$ we get a polynomial in which $c b a$ enters with the coeficient

$$
\begin{aligned}
& (-1)^{\hat{a} \hat{d}+\hat{b} \hat{c}}\left(q_{v}^{-1} q_{h}-q_{v} q_{h}^{-1}\right)\left(q_{v}^{-1} q_{h}^{-1}-q_{v} q_{h}\right)\left(q_{h}^{-1}+q_{h}\right)^{-2} \\
& +(-1)^{\hat{b} \hat{c}+\hat{a} \hat{c}+\hat{a} \hat{b}} q_{v}^{(-1)^{\hat{k}+1}} q_{h}^{(-1)^{\hat{i}+1}}\left(q_{v}^{-1}+q_{v}\right)\left(q_{h}^{-1}+q_{h}\right)^{-1} .
\end{aligned}
$$

On the other hand, $c b a$ appears in $R_{12} R_{23} R_{12}(a b c)$ with the coefficient which coincides with the second term of (5.7). Therefore, the two normal forms can coincide only if either $q_{v}^{-1} q_{h}=q_{v} q_{h}^{-1}$ or $q_{v}^{-1} q_{h}^{-1}=q_{v} q_{h}$, i.e., $q_{v}= \pm q_{h}^{ \pm 1}$. Then one checks that in this condition, the coefficients of $d a^{2}$ in these two normal forms automatically coincide for $\hat{a}=0$; otherwise, $d a^{2}=0$.

5.6. Uniqueness of the Normal Form. Assume now that normal cubic monomials are linearly independent in $E_{q}$. We want to deduce that monomials of any degree are linearly independent. This is equivalent to the statement that they have a unique normal form.

There is a well known combinatorial principle allowing us to ascertain such uniqueness.

Namely, let $p$ be a polynomial and $T^{\prime}, T^{\prime \prime}$ two elementary transformations applicable to $p$. Suppose that there exist two sequences of elementary transformations $S_{1}^{\prime}, \ldots, S_{a}^{\prime}$ and $S_{1}^{\prime}, \ldots, S_{b}^{\prime}$ such that

$$
S_{a}^{\prime} \ldots S_{1}^{\prime} T^{\prime}(p)=S_{b}^{\prime \prime} \ldots S_{1}^{\prime \prime} T^{\prime \prime}(p) .
$$

If this condition is valid for all triples $\left(p, T^{\prime}, T^{\prime \prime}\right)$ then the normal form is unique.

Let us check this condition in our case. In fact, if $T^{\prime}, T^{\prime \prime}$ are either applied to different monomials in $p$, or to the same monomial but the respective pairs do not intersect, we can simply put $S_{1}^{\prime}=T^{\prime \prime}, S_{1}^{\prime \prime}=T^{\prime}$. Otherwise, up to renaming, $T^{\prime}$ replaces $u v$ and $T^{\prime \prime}$ replaces $v w$ in a neighbouring triple $u v w, u>v>w$. By assumption, $u v w$ has a unique normal form. Reducing $T^{\prime}(u v)$ and $T^{\prime \prime}(v w)$ to this common normal form we obtain $S_{i}^{\prime}$ and $S_{j}^{\prime \prime}$.

5.7. The $n=2$ case. Here $q_{v}=q_{h}$, so that Lemma 5.5 can be directly applied. No more cubic monomials should be checked.

\section{References}

1. Abe, E.: Hopf algebras. Cambridge Tracts in Math., Vol. 74. Cambridge: Cambridge University Press 1980

2. Drinfeld, V.G.: Quantum groups. Proc. Int. Congr. Math., Berkeley 1, 798-820 (1986) 
3. Faddeev, L.D., Reshetikhin, N.Y., Takhtajan, L.A.: Quantization of Lie groups and Lie algebras. Preprint LOMI, 1987

4. Jimbo, M.: A $q$-analogue of $U(g l(N+1))$, Hecke algebra and the Yang-Baxter equation. Lett. Math. Phys. 11, 247-252 (1986)

5. Manin, Yu.I.: Some remarks on Koszul algebras and quantum groups. Ann. Inst. Fourier, Tome XXXVII, F.4, 191-205 (1987)

6. Manin, Yu.I.: Quantum groups and non-commutative geometry. Preprint Montreal University, CRM-1561, 1988

7. Woronowicz, S.L.: Compact matrix pseudogroups. Commun. Math. Phys. 111, 613-665 (1987)

Communicated by A. Jaffe

Received December 11, 1988

Note added in proof. John Tate has independently obtained the Theorem 1.14 (for the pure even format). E. Demidov has calculated the necessary and sufficient conditions for cubic monomials to be independent. They run as follows: if

$$
q_{i j}=\varepsilon_{i j} q^{v_{i j}}, \quad \varepsilon_{\imath j}= \pm 1, \quad v_{i j}= \pm 1
$$

then

$$
\left(v_{i J}-v_{l k}\right)\left(v_{j k}-v_{i k}\right)=0
$$

for all $i<j<k$. It follows that, after a renumbering of $x_{1}, \ldots, x_{n}$, one can assume that $v_{i j}$ do not depend on $(i j)$. 
